\title{
Dispneia Ansiosa e Dispneia por COVID-19: um Diagnóstico Diferencial
}

\section{Anxious Dyspnea and COVID-19 Dyspnea: a Differential Diagnosis}

\author{
Eduarda Tashikawa ${ }^{1}$ \\ Bruna Ramos ${ }^{2}$ \\ Karina Martins ${ }^{3}$
}

Alfredo Simonetti ${ }^{4}$

\section{RESUMO:}

A dispneia é um sintoma em comum entre a COVID-19 e os quadros ansiosos, o que abre espaço para dúvidas no diagnóstico entre as duas síndromes. Esse estudo tem como objetivo identificar características que auxiliem nessa diferenciação. Foi realizada uma pesquisa bibliográfica na base de dados PUBMED, utilizando os MESH terms 'anxiety', 'dyspnea', 'COVID-19', 'SARS-CoV-2', totalizando um total de 658 artigos, que após a aplicação de critérios de inclusão e exclusão foram selecionados um total de 15 artigos para a realização da revisão. As duas patologias apresentaram possíveis mecanismos fisiopatológicos diferentes. Na COVID-19, a dispneia está associada ao aumento dos receptores ECA2 nas células epiteliais alveolares além de outros locais, enquanto na ansiedade o mecanismo parece estar associado ao sistema simpático que causa a tensão muscular respiratória e consequente sensação de dispneia, além da ativação mais intensa e prolongada das áreas cerebrais associadas ao medo frente uma ameaça respiratória. Os sintomas mais frequentes na COVID-19 foram febre, tosse e alterações no olfato, juntamente de alterações na tomografia de tórax, como o padrão em vidro fosco. Já na ansiedade, a sensação de falta de ar pode acontecer mesmo na ausência de hipoxemia. Portanto, para diferenciar os dois quadros, a presença de outros sintomas como febre, tosse e anosmia, além de alterações na tomografia de tórax e a presença de hipoxemia são fatores que podem auxiliar.

Palavras-chave: Dispneia, Ansiedade, COVID-19, Sars-Cov-2

\section{ABSTRACT:}

Dyspnea is a common symptom between COVID-19 and anxiety disorders, which leaves room for doubts in the diagnosis between the two syndromes. This study aims to identify characteristics that help in this differentiation. A literature search was carried out in the PUBMED database, using the MESH terms 'anxiety', 'dyspnea', 'COVID-19', 'SARS-CoV-2', totaling 658 articles, which after applying the criteria for inclusion and exclusion, a total of 15 articles were selected for the review. The two pathologies presented different possible pathophysiological mechanisms. In COVID-19, dyspnea is associated with increased ACE2 receptors in alveolar epithelial cells in addition to other sites, while in anxiety the mechanism seems to be associated with the sympathetic system that causes respiratory muscle tension and consequent dyspnea sensation, in addition to activation more intense and prolonged of the brain areas associated with fear in the face of a respiratory threat. The most frequent symptoms in COVID-19 were fever, cough and changes in smell, together with changes in chest tomography, such as the ground-glass pattern. While in anxiety, the feeling of shortness of breath can happen even in the absence of hypoxemia. Therefore, to differentiate the two conditions, the presence of other symptoms such as fever, cough and anosmia, in addition to changes in the chest tomography and the presence of hypoxemia are factors that can help.

Keywords: Dyspnea, Anxiety, COVID-19, Sars-Cov-2

\footnotetext{
${ }^{1}$ Graduanda do Curso de Medicina do Centro Universitário São Camilo. Email: eduardaaoi@outlook.com

${ }^{2}$ Graduanda do Curso de Medicina do Centro Universitário São Camilo. Email: bruna.cassia.ramos@aluno.saocamilosp.br

${ }^{3}$ Graduanda do Curso de Medicina do Centro Universitário São Camilo. Email: karina.martins@aluno.saocamilo-sp.br

${ }^{4}$ Docente do Curso de Medicina do Centro Universitário São Camilo. Email: alfredo.simonetti@prof.saocamilo-sp.br
} 


\section{INTRODUÇÃO}

A COVID-19 é uma doença de distribuição mundial, de rápida propagação, com capacidade de transmissão maior comparada à outras síndromes respiratórias causadas por vírus da mesma família, como a Síndrome Respiratória Aguda Grave, e a Síndrome Respiratória do Oriente Médio [15]. Dentre os sintomas mais frequentes da COVID-19, a dispneia, estava presente em 30,82\% dos pacientes e é descrita como um fator que pode indicar maior gravidade da doença [3]. Entretanto, a falta de ar não é exclusiva dessa patologia, estando presente também nos distúrbios ansiosos.

A falta de ar é um sintoma subjetivo, como resultado da combinação de múltiplos fatores (fisiológicos, psicológicos, sociais e ambientais), portanto a dispneia não reflete apenas o fisiológico do indivíduo, mas também pode refletir o seu psicológico. Desse modo, pacientes com comorbidades psicológicas, principalmente aqueles com transtornos afetivos, como a ansiedade, comumente podem apresentar dispneia [14].

Essa convergência da presença da falta de ar em ambas as síndromes, COVID-19 e ansiedade, abre espaço para dúvidas no diagnóstico etiológico do sintoma e consequente dúvida na abordagem terapêutica, visto que cada patologia tem uma indicação terapêutica diferente [15], [14]. A fim de auxiliar na resolução dessas dúvidas, essa revisão tem como objetivo identificar características dessas duas patologias que irão auxiliar nas suas diferenciações.

\section{METODOLOGIA}

Para a realização desta revisão narrativa foram realizadas 2 pesquisas utilizando o mecanismo de MESH terms na plataforma PUBMED no dia 12 de agosto de 2021. A primeira pesquisa foi ("COVID-19"[Mesh] OR "SARS-CoV-2"[Mesh]) AND "Dyspnea"[Mesh] totalizando um total de 390 artigos. A segunda pesquisa foi ("Anxiety"[Mesh]) AND "Dyspnea"[Mesh] totalizando um total de 268 artigos. Em ambas foi aplicado o filtro para estudos publicados nos últimos 10 anos. Em seguida, esses 658 artigos foram analisados pelo título, tipo do artigo e resumo. Foram excluídos artigos repetidos, artigos que não se adequaram ao tema e relatos de caso, totalizando um total de 15 artigos incluídos no trabalho.

\section{RESULTADOS}

Os resultados encontrados encontram-se na tabela abaixo.

\begin{tabular}{|c|c|c|}
\hline Título & Ano & Conclusão \\
\hline $\begin{array}{l}1 \text { Diagnostic accuracy of } \\
\text { subjective dyspnoea in } \\
\text { detecting hypoxaemia } \\
\text { among outpatients with } \\
\text { COVID-19: a retrospective } \\
\text { cohort study }\end{array}$ & 2021 & $\begin{array}{l}\text { O estudo mostra que cerca de } 20 \% \text { dos pacientes com } \\
\text { COVID-19 apresentam dispneia, a qual é comum ser } \\
\text { associada à gravidade da doença. Os casos fatais, além } \\
\text { de manifestarem dispneia, também demonstraram } \\
\text { menores saturações de oxigênio e maiores complicações } \\
\text { como a síndrome do desconforto respiratório agudo. Foi } \\
\text { vista a limitação da utilidade da dispneia na detecção da } \\
\text { hipoxemia com sensibilidade de } 47 \% \text { e especificidade } \\
\text { de } 80 \% \text { É possível a presença da dispneia mesmo na } \\
\text { ausência da hipoxemia como nos casos vistos em que o } \\
\text { indivíduo apresenta altos níveis de ansiedade. }\end{array}$ \\
\hline
\end{tabular}


2 Pulmonary function and functional capacity in COVID-19 survivors with persistent dyspnoea

3 Clinical manifestations of COVID-19 in the general population: systematic review
4 Breathlessness and the body: neuroimaging clues for the inferential leap
A dispneia é considerada um preditor independente de morbidade e de mortalidade, sendo relacionada à redução da capacidade funcional. Foi relatada uma disparidade racial e étnica em relação a infecção e hospitalização por COVID-19, além de maiores taxas de comorbidades como diabetes, obesidade e até mesmo associação entre as comorbidades que aumentam a gravidade desses pacientes.

O estudo demonstrou que a dispneia, portanto, é um sintoma persistente devido às anormalidades apresentadas por esses pacientes - como capacidade funcional reduzida, aumento da dessaturação e esforço durante a TC - e que está presente nos mais variados graus de gravidade da COVID-19.

2021 O estudo relatou que os sintomas mais frequentes entre os pacientes com COVID-19 foram: febre (57,93\%), tosse $(54,21 \%)$, e dispneia $(30,64 \%)$. Esses 3 sintomas faziam parte da tríade de sintomas da COVID-19, que posteriormente o Centro de Controle e Prevenção de Doenças dos Estados Unidos (CDC) acrescentou a lista: calafrios, dores musculares, dores de cabeça, dor de garganta e perda de paladar ou olfato. A dispneia foi relacionada à maior gravidade da doença. A maior parte dos pacientes graves apresentavam no exame físico: dispneia, aumento da frequência respiratória, tremor de fala, murmúrios vesiculares diminuídos e embotamento à percussão pulmonar. Nos achados radiológicos desse grupo foram vistos opacidades em vidro fosco e consolidação, principalmente na periferia. Pacientes assintomáticos e sem achados radiológicos compuseram $2,27 \%$ do estudo, composto principalmente de jovens e mulheres, apesar da porcentagem pequena, a ausência de sintomas apresenta uma dificuldade no rastreio da doença.

2017 A estrutura bayesiana representa a percepção final de falta de ar por um conjunto de valores prováveis. Ele visa minimizar erros de predição das informações sensoriais aferentes em relação às expectativas anteriores.

As previsões interoceptivas ocorrem no interior das camadas profundas do córtex cingulado, anterior e na ínsula anterior que são agranulares, cujos neurônios de projeção terminal nos córtex granulares como o córtex sensorial primário e a ínsula posterior.

A sensibilidade à ansiedade pode interagir com a precisão interoceptiva e detecção de ameaças, além de catastrofização, o que desloca a atenção e altera a importância das informações sensoriais. 
5 Reduced neural gating of respiratory sensations is associated with increased dyspnoea perception
6 COVID-19: comparative

clinical features and outcome in 114 patients with or without pneumonia (Nord Franche-Comte Hospital, France)
No experimento aplicado nesse estudo, a antecipação da falta de ar se relacionou inversamente com o córtex précuneiforme (autorreflexão e recuperação da memória), o que mostra que indivíduos altamente sensíveis à ansiedade dependam menos de experiências anteriores para a percepção interoceptiva. Ademais, houve maior atividade da ínsula anterior (o que pode explicar erros de previsão) e menor da ínsula posterior e córtex sensorial primário (modulação negativa do ganho de informação aferente).

Sendo assim, elucida-se a menor confiança em episódios anteriores para determinar as percepções e torna esse sistema bayesiano menos desenvolvido.

2018

A respiração, em condições normais, ocorre sem que a consciência seja atingida, sendo, portanto, automática.

Isso faz com que o processamento neural seja direcionado para as atividades diárias e não se fixe em sensações respiratórias irrelevantes.

A ansiedade está associada a redução desse bloqueio neural, mecanismo que gera uma superpercepção da dispneia e redução gating neural de sensações respiratórias sob níveis crescentes de carga. Além disso, pode estar parcialmente relacionada aos processos de atenção.

2020 Pacientes com apresentações mais graves de COVID-19 como pneumonia apresentavam uma média de idade de 68 anos e eram do sexo masculino. Dos pacientes com pneumonia, $72 \%$ eram portadores de comorbidades como doença cardiovascular, diabetes mellitus, DPOC e neoplasia. Ademais, o curso da pneumonia pode resultar em insuficiência respiratória e em SDRA.

Os sintomas iniciais desses indivíduos foram febre ou sensação de febre e dispneia e, apenas $28 \%$ desses, apresentavam anosmia.

O receptor funcional do vírus da COVID-19, a proteína ACE2, se expressa amplamente nas células epiteliais alveolares, enterócitos e células endoteliais, incluindo no SNC. Com isso, explicam-se os sintomas de dispneia, tosse e crepitação na ausculta pulmonar nos pacientes graves.

O estudo também demonstrou que a distribuição parcial desses receptores de entrada pode explicar parcialmente a diferença de sintomas entre as apresentações graves e leves, haja vista que, neste último grupo, a maior prevalência é no sexo feminino e há a presença de anosmia, disgeusia, cefaleia, dor de garganta, rinorreia e obstrução nasal. 
7 Neural responses to affective pictures while anticipating and perceiving respiratory threat
8 Symptoms, symptom relief and support in COVID-19 patients dying in hospitals during the first pandemic wave

9 Clinical profile and factors associated with COVID-19 in Yaounde, Cameroon: A prospective cohort study
2016

A falta de ar é resultado de uma resposta fisiológica pronunciada em relação ao medo. A antecipação da falta de ar pode desempenhar um papel crucial na desordem do sistema respiratório e da ansiedade. Tal mecanismo é modulado pela sensibilidade do indivíduo à ansiedade que impacta o processamento de estímulos emocionais e está relacionado a sinais de ameaça respiratória como a falta de ar.

Como visto no estudo, indivíduos altamente ansiosos se concentram mais na estimulação ameaçadora do corpo o que gera um resultado direto na diminuição da capacidade neural para o processamento das imagens apresentadas. Sendo assim, exibiram um estado de hipervigilância durante a percepção da falta de ar e, com isso, redução da atenção para estímulos negativos das imagens apresentadas logo no início do experimento.

2021

O estudo demonstrou que pacientes acometidos pela COVID-19 eram, em sua maioria, do sexo masculino e possuíam uma média de idade superior. Além disso, a presença da falta de ar em pacientes que vieram a óbito pela COVID-19 era maior entre os mais jovens e que a velhice é um fator preditivo para menos sintomas respiratórios, portanto, mais inespecíficos.

2021

A dispneia é um dos sintomas detectados na infecção por SARS-CoV-2, neste estudo $49,8 \%$ apresentaram dispneia. Foram vistos alguns preditores de gravidade para COVID-19 como sexo masculino, idade avançada, comorbidades (diabetes, HIV e doenças pulmonares), presença de fadiga, e inclusive a presença de dispneia.

10 Spring 2020 COVID-19

2021

Surge: Prospective Relations between Demographic

Factors, Personality Traits, Social Cognitions and Guideline Adherence, Mask Wearing, and Symptoms in a U.S. Sample
O estudo pesquisou a associação prospectiva entre os traços de personalidade, crenças e intenções relacionadas às diretrizes e à adesão delas, uso de máscaras, contagem de sintomas e teste viral para SARS-CoV-2 durante o primeiro surto de casos e mortes nos EUA em 2020.

Houve maior neuroticismo, ou seja, menor estabilidade emocional e maior reatividade ao estresse na pesquisa apresentada pelo estudo. Com isso, houve maior contagem de sintomas de acompanhamento e maior tendência ansiosa e instável na percepção e no relato das queixas psicossomáticas. Ademais, tal fato foi consistente com as evidências que mostravam maior neuroticismo associado a mais problemas de saúde. 
11 Respiratory Muscle

Tension as Symptom

Generator in Individuals

With High Anxiety

Sensitivity
12 Diagnostic accuracy of symptoms as a diagnostic tool for SARS-CoV 2 infection: a cross-sectional study in a cohort of 2,173 patients
13 Relationship of

sociodemographics, comorbidities, symptoms and healthcare access with early COVID-19 presentation and disease severity
2013 Este estudo relatou que indivíduos altamente sensíveis à ansiedade são sensíveis à tensão dos músculos respiratórios os quais contribuem para a percepção de dispneia, mais especificamente o músculo peitoral, resultando em mudança do padrão respiratório e hiperinsuflação dinâmica, responsável pela indução de dispneia e fadiga em casos crônicos.. Além disso, houve um aumento de sintomas associados com hiperventilação e falta de ar durante tensão do músculo torácico.

Também sugeriu que alterações de PCO2 não são necessárias para que haja a produção de sensações intensas de dispneia. Por fim, sintomas classicamente associados à hipocapnia foram gerados pela tensão muscular respiratória nesses indivíduos.

Desse modo, explica-se a ligação entre ansiedade e níveis elevados de tensão muscular respiratória por meio dos efeitos gerados pelo sistema nervoso simpático.

2021 O estudo demonstrou uma relação estreita da infecção por SARS-CoV-2 com a presença de anosmia, febre, dispneia e tosse, o que permite maior acurácia no diagnóstico presuntivo.

Ademais, a presença de dispneia e hipóxia ocorrem devido a lesão pulmonar causada pelo vírus. Isso se deve a alta expressão de receptores ECA2 nas células apicais pulmonares, os quais promovem a adesão, penetração e destruição do tecido pulmonar, produção de exsudato inflamatório intersticial e alveolar além de edema.

Em relação a anosmia, há três possíveis rotas para a invasão do sistema nervoso pelo vírus, como a via hematogênica, por meio da placa cribriforme através do epitélio do nervo olfatório e um transporte axonal retrógrado para o SNC. Por outro lado, também foi considerada a possibilidade de o vírus causar aumento das bradicininas e ativação de receptores dos centros responsáveis pelo paladar e olfato na medula oblonga, o que também explicaria tais apresentações clínicas.

Sendo assim, tais mecanismos fisiopatológicos e seus respectivos sintomas contribuem para o diagnóstico dessa patologia.

2020 O estudo relata maior incidência de homens, idade maior de 65 anos, falta de ar entre os pacientes internados, além de associação da gravidade da doença com a presença de falta de ar, etnia asiática e hipertensão arterial. 
14 Affective traits, states, and breathlessness

2020

O estudo menciona a dispneia como uma experiência subjetiva, resultante de uma combinação de fatores fisiológicos, psicológicos, sociais e ambientais. $\mathrm{O}$ afeto negativo está tipicamente relacionado a níveis maiores de falta de ar. A dispneia se demonstrou frequente entre os indivíduos com traços de personalidade negativa não específica, principalmente ansiedade e depressão. Essa frequência está presente tanto em grupos de indivíduos saudáveis quanto em indivíduos com alguma outra patologia (DPOC, Doença de Parkinson, câncer, doenças cardiorrespiratórias). Dois estudos experimentais em população saudável mostraram que a falta de ar induzida por carga resistiva gerou estados afetivos negativos, incluindo medo, desconforto, sintomas de pânico e comportamento evasivo. Esses sintomas se intensificaram com a adição de exclusão social no estudo e a ameaça iminente de dispneia, a sensação de falta de ar aumentou principalmente em indivíduos com traço de ansiedade. Outros estudos experimentais apontaram que o estado afetivo positivo, obtido através de estimulação musical agradável e sensação de inclusão social através de jogos virtuais, teve relação com a melhora da dispneia em população saudável.

15 Epidemiologic, clinical, and laboratory findings of the COVID-19 in the current pandemic: systematic review and meta-analysis
2020

$\mathrm{O}$ estudo mostrou que os sintomas mais comuns em pacientes com COVID-19 são: febre (76,70, IC 95\%: 64,86-85,44\%), tosse $(67,76$, IC 95\%: 60,06-74,61\%) e sintomas olfatórios $(44,40 \%)$. A dispneia teve uma frequência de 37,49\%. A dispneia e a anorexia apresentaram uma frequência maior em pacientes em condições graves, sendo a dispneia o sintoma mais comum em pacientes graves nos Estados Unidos. Na tomografia computadorizada, a maioria dos pacientes com COVID-19 tinha opacidade em vidro fosco, com um envolvimento pulmonar bilateral mais comum nas fases intermediárias e tardias da doença, com o aparecimento de consolidações no terceiro estágio da doença (entre 1 e 2 semanas), podendo aparecer também nessa fase alterações intersticiais indicativas de fibrose.

\section{DISCUSSÃO}

Durante o período da pandemia de COVID-19 em 2020, nos EUA, mais especificamente no surto de casos e mortes, houve um aumento do neuroticismo, o que culminou em maior instabilidade emocional dos indivíduos pesquisados. Mostrou-se que ocorreu um aumento na contagem de sintomas de acompanhamento, juntamente com maior tendência ansiosa e aumento dos relatos das queixas psicossomáticas. Evidenciou-se que o aumento do neuroticismo está associado ao aumento de problemas de saúde [10].

Como houve um provável viés dos sintomas relatados devido à influência emocional, o presente trabalho visou identificar características clínicas (propedêuticas e anamnésticas) e 
fisiopatológicas que permitam diferenciar a dispneia ansiosa da dispneia por COVID-19.

No que tange à similaridade da apresentação clínica da COVID-19 e da tendência ansiosa, destacamos a falta de ar. Apesar da dispneia ser um sintoma comum entre a COVID-19 e a ansiedade, os seus mecanismos fisiopatológicos demonstraram-se diferentes. Na infecção por SARS-CoV-2, a dispneia ocorre devido à lesão pulmonar. A presença do vírus aumenta a expressão de receptores ECA2 nas células apicais pulmonares, o que promove a adesão, penetração e destruição do tecido pulmonar, além da formação de exsudato inflamatório intersticial, alveolar e edema [6], [12].

Já na ansiedade, a sensação de falta de ar está associada à redução do bloqueio neural da percepção da respiração. Essa redução do bloqueio faz com que o processamento neural seja direcionado para sensações respiratórias irrelevantes, o que gera uma super percepção da dispneia [5]. Além disso, a ansiedade pode interagir com a precisão interoceptiva nas camadas profundas do córtex e detecção de ameaças e catastrofização, provocando o deslocamento da atenção em indivíduos ansiosos e a alteração da importância das informações sensoriais [4]. Indivíduos ansiosos também apresentam maior sensibilidade à tensão muscular respiratória. Essa tensão muscular, especificamente do músculo peitoral, altera o padrão respiratório, podendo causar hiperinsuflação e indução da dispneia e fadiga crônica. Tais alterações foram mais associadas a sintomas de hipocapnia, ao invés de hipoxemia, nesse grupo de indivíduos [11].

Assim, essa diferença fisiopatológica dos dois quadros pode auxiliar no seu diagnóstico diferencial. $\mathrm{Na}$ ansiedade, a dispneia pode não vir acompanhada de hipoxemia, por estar relacionada a uma hiperpercepção das sensações respiratórias e aumento da tensão muscular respiratória, podendo estar mais associada a sintomas de hipocapnia [5], [11]. Enquanto na COVID-19, a falta de ar pode vir associada à hipoxemia, por ser causada por uma lesão pulmonar [12]. Todavia, a ausência de hipoxemia não exclui a possibilidade de ser infecção por SARS-Cov-2, já que a dispneia pode vir na ausência de hipoxemia nesse grupo, como foi visto nos casos em que esses indivíduos com COVID-19 apresentavam altos níveis de ansiedade [1].

Febre e tosse fizeram parte dos sintomas mais frequentes em pacientes com COVID-19 nos artigos analisados. A combinação desses dois sintomas, com a adição da dispneia, foi descrita como tríade de sintomas da COVID-19 pelo Centro de Controle e Prevenção de Doenças dos Estados Unidos [3], [12]. Sintomas olfatórios também apresentaram uma incidência considerável de $44,40 \%$. A fisiopatologia da anosmia parece estar relacionada com a via de infecção do vírus para o Sistema Nervoso Central, que uma das rotas é pela placa cribriforme através do epitélio do nervo olfatório [15], [12]. Desse modo, presença de febre, tosse ou anosmia além da dispneia pode auxiliar no diagnóstico da COVID-19.

Na COVID-19, a dispneia está associada a quadros mais graves da doença, sendo considerada um preditor independente de morbimortalidade [1], [2], [9], [13], [15]. No exame físico dos pacientes graves, estavam presentes o aumento da frequência respiratória, a dispneia, o tremor de fala, murmúrios vesiculares diminuídos, presença de crepitação na ausculta pulmonar e embotamento à percussão pulmonar [3], [6]. A presença dessas alterações do exame físico pulmonar pode auxiliar na diferenciação entre a dispneia ansiosa e a dispneia por COVID-19.

Outros fatores de gravidade da COVID-19 foram: sexo masculino, idade avançada e presença de comorbidades, como DPOC, diabetes mellitus, e doenças cardiovasculares [2], [6], [8], [9], [13]. Entretanto, a presença de comorbidades também está relacionada à dispneia ansiosa. A falta de ar demonstrou-se frequente na população com traços de personalidade negativa e com alguma comorbidade que pode afetar a capacidade respiratória do paciente, como DPOC, provavelmente devido ao medo da ameaça iminente de dispneia, o que intensifica o sintoma. Esse mecanismo também foi observado em um experimento em indivíduos saudáveis expostos a carga resistiva, que acabaram desenvolvendo a sensação de falta de ar [7], [14]. Como esses fatores não se demonstraram específicos da COVID-19, além da falta de dados para melhor comparação, eles não foram considerados como características que auxiliariam na diferenciação das duas patologias.

Em contrapartida, alterações vistas em exames de imagem podem auxiliar a predileção do diagnóstico de COVID-19 em comparação a um quadro ansioso. Na tomografia, a maioria dos 
pacientes com COVID-19 apresentavam opacidade em vidro fosco, com envolvimento bilateral, e a presença de consolidação após a primeira semana de infecção [15]. Os achados radiológicos como vidro fosco e a presença de consolidação na periferia já não são encontrados em casos de transtornos ansiosos [3].

\section{CONCLUSÃO}

Tendo em vista as diferenças fisiopatológicas entre a dispneia ansiosa e por COVID-19 e a importância do diagnóstico diferencial desse sintoma, deve-se considerar a presença de sinais e sintomas associados como anosmia, febre, tosse e a presença ou não de hipoxemia. Além de avaliar achados radiológicos e tomográficos, como o vidro fosco e consolidação pulmonar, juntamente com alterações encontradas no exame físico pulmonar, como presença de crepitação na ausculta e embotamento pulmonar na percussão, a fim de melhor distingui-las a nível de pronto atendimento.

\section{REFERÊNCIAS}

1. BEREZIN, Linor et al. Diagnostic accuracy of subjective dyspnoea in detecting hypoxaemia among outpatients with COVID-19: a retrospective cohort study. Bmj Open. Toronto, p. 1-9. mar. 2021. Disponível em: https://bmjopen.bmj.com/content/11/3/e046282.long. Acesso em: 23 ago. 2021.

2. CORTÉS-TELLES, Arturo et al. Pulmonary function and functional capacity in COVID-19 survivors with persistent dyspnoea. Respiratory Physiology \& Neurobiology. Yucatan, p. 1-4. fev. 2021. Disponível em: https://www.sciencedirect.com/science/article/abs/pii/S156990482100029X?via \%3Dihub. Acesso em: 21 ago. 2021.

3. da Rosa Mesquita $\mathrm{R}$ et al. Clinical manifestations of COVID-19 in the general population: systematic review. Wiener klinische Wochenschrift. 2021 Apr; 133(7-8):377-382.

4. FAUll, Olivia K.; HAYEN, Anja; PATTINSON, Kyle T.s.. Breathlessness and the body: neuroimaging clues for the inferential leap. Cortex, [S.L.], v. 95, p. 211-221, out. 2017. Elsevier BV.

5. HERZOG, Michaela; SUCEC, Josef; VAN DIEST, Ilse; BERGH, Omer van Den; CHAN, PeiYing Sarah; DAVENPORT, Paul; VON LEUPOLDT, Andreas. Reduced neural gating of respiratory sensations is associated with increased dyspnoea perception. European Respiratory Journal, [S.L.], v. 52, n. 1, p. 1800559, 17 maio 2018. European Respiratory Society (ERS).

6. JULIETTEKADIANE-OUSSOU, N'dri et al. COVID-19: comparative clinical features and outcome in 114 patients with or without pneumonia (Nord FrancheComte Hospital, France). Microbes And Infection. Trevenans, p. 622-625. nov. 2020. Disponível em: https://www.sciencedirect.com/science/article/abs/pii/S1286457920301805?via \%3Dihub. Acesso em: 21 ago. 2021.

7. JURAVLE, Georgiana; REICHERTS, Phillipp; RIECHMANN-WEINSTEIN, Mirjam; WIESER, Matthias J.; VON LEUPOLDT, Andreas. Neural responses to affective pictures while anticipating 
and perceiving respiratory threat. Psychophysiology, [S.L.], v. 54, n. 2, p. 182-192, 21 out. 2016. Wiley. http://dx.doi.org/10.1111/psyp.12776.

8. MARTINSSON, Lisa; BERGSTRÖM, Jonas; HEDMAN, Christel; STRANG, Peter; LUNDSTRÖM, Staffan. Symptoms, symptom relief and support in COVID-19 patients dying in hospitals during the first pandemic wave. Bmc Palliative Care, [S.L.], v. 20, n. 1, p. 1-23, 1 jul. 2021. Springer Science and Business Media LLC.

9. MBARGA, Nicole Fouda et al. Clinical profile and factors associated with COVID-19 in Yaounde, Cameroon: A prospective cohort study. Plos One. Yaounde, p. 1-12. maio 2021. Disponível em: https://journals.plos.org/plosone/article/authors?id=10.1371/journal.pone.02515 04. Acesso em: 21 ago. 2021.

10. MILAD, Elizabeth; BOGG, Tim. Spring 2020 COVID-19 Surge: Prospective Relations between Demographic Factors, Personality Traits, Social Cognitions and Guideline Adherence, Mask Wearing, and Symptoms in a U.S. Sample. Annals Of Behavioral Medicine. Detroit, p. 665-676. maio 2021. Disponível em: https://academic.oup.com/abm/article/55/7/665/6276221. Acesso em: 23 ago. 2021.

11. RITZ, Thomas; MEURET, Alicia E.; BHASKARA, Lavanya; PETERSEN, Sibylle. Respiratory Muscle Tension as Symptom Generator in Individuals With High Anxiety Sensitivity. Psychosomatic Medicine, [S.L.], v. 75, n. 2, p. 187-195, fev. 2013. Ovid Technologies (Wolters Kluwer Health).

12. ROMERO-GAMEROS, Carlos Alfonso; COLIN-MARTÍNEZ, Tania; WAIZELHAIAT, Salomón; VARGAS-ORTEGA, Guadalupe; FERAT-OSORIO, Eduardo; GUERRERO-PAZ, José Alberto; INTRIAGO-ALOR, Marielle; LÓPEZMORENO, Mayra Alejandra; CUEVAS-GARCÍA, Carlos Fredy; MENDOZAZUBIETA, Victoria. Diagnostic accuracy of symptoms as a diagnostic tool for SARS-CoV 2 infection: a cross-sectional study in a cohort of 2,173 patients. Bmc Infectious Diseases, [S.L.], v. 21, n. 1, p. 1-24, 11 mar. 2021. Springer Science and Business Media LLC.

13. VAUGHAN, Laura; VERUTTIPONG, Darlene; SHAW, Jonathan G.; LEVY, Noemie; EDWARDS, Lauren; WINGET, Marcy. Relationship of sociodemographics, comorbidities, symptoms and healthcare access with early COVID-19 presentation and disease severity. Bmc Infectious Diseases, [S.L.], v. 21, n. 1, p. 1-19, 9 jan. 2021. Springer Science and Business Media LLC.

14. von Leupoldt A, Denutte Y. Affective traits, states, and breathlessness. Current opinion in supportive and palliative care. 2020 Sep;14(3):182-189.

15. Xie $\mathrm{Y}$ et al. Epidemiologic, clinical, and laboratory findings of the COVID-19 in the current pandemic: systematic review and meta-analysis. BMC infectious diseases. 2020 Aug; 20(1):640. 